\title{
Developing indicators for measuring low-value care: mapping Choosing Wisely recommendations to hospital data
}

\author{
Kelsey Chalmers ${ }^{1,2^{*}+}$ (D), Tim Badgery-Parker ${ }^{1,2^{*}+}$ (D), Sallie-Anne Pearson ${ }^{3}$ (D), Jonathan Brett ${ }^{3}$, Ian A. Scott ${ }^{4,5}$ (D) \\ and Adam G. Elshaug ${ }^{1}$ (D)
}

\begin{abstract}
Objective: Low-value health care refers to interventions where the risk of harm or costs exceeds the likely benefit for a patient. We aimed to develop indicators of low-value care, based on selected Choosing Wisely (CW) recommendations, applicable to routinely collected, hospital claims data.

Results: We assessed 824 recommendations from the United States, Canada, Australia and the United Kingdom CW lists regarding their capacity to be measured in administrative hospital admissions datasets. We selected recommendations if they met the following criteria: the service occurred in the hospital setting (observable in setting); a claim recorded the use of the service (record of service); the appropriate/inappropriate use of the service could be mapped to information within the hospital claim (indication); and the service is consistently recorded in the claims (consistent documentation). We identified 17 recommendations (15 services) as measurable. We then developed low-value care indicators for two hospital datasets based on the selected recommendations, previously published indicators, and clinical input.
\end{abstract}

Keywords: Inappropriate care, Low-value care, Quality measurement, Choosing Wisely, Hospitals, Quality of health care, Disinvestment

\section{Introduction}

Low-value care offers limited or no benefit and poses unnecessary risks and costs to patients $[1,2]$. Publishing "do-not-do" recommendations, which detail inappropriate services for patient groups, is a recent strategy to redress low-value care. An example of this is the international Choosing Wisely (CW) campaign [3].

\footnotetext{
*Correspondence: kelsey.chalmers@sydney.edu.au; tim.

badgeryparker@sydney.edu.au

${ }^{\dagger}$ Kelsey Chalmers and Tim Badgery-Parker are co-first authors and contributed equally to this work

${ }^{1}$ Menzies Centre for Health Policy, School of Public Health, Charles

Perkins Centre, University of Sydney, Sydney, NSW 2006, Australia

Full list of author information is available at the end of the article
}

One way to assess low-value care is to develop direct measures in routinely collected data [4]. These datasets, including health insurance claims and hospital administrative databases, provide population-level insights on service utilisation. Direct measures of low-value care use information collected at the individual patient level to distinguish inappropriate from appropriate care [5]. This approach contrasts with measuring low-value care indirectly via geographic variation in services, which does not necessarily distinguish unwarranted from warranted variation. Using these direct measures is an additive approach to measuring low-value care, as described by Miller et al. [6], and is suitable for both patient- and service-centric measures of low-value care [7].

We isolated $\mathrm{CW}$ recommendations with characteristics conducive to indicator development, thus allowing for direct measurement in routinely collected data on inpatient admissions. While these indicators are not formal 
quality metrics, which would require further investigation of their reliability and validity, they can provide an estimate of low-value services used within health care systems.

\section{Main text \\ Methods \\ Datasets}

Our two datasets were (1) hospital and medical claims from a group of Australian private health insurance (PHI) funds, and (2) public hospital admissions data from Australia's most populous state (New South Wales). Australians can receive care in public or private hospitals, and care is funded by some combination of patients' out-of-pocket payments, their PHI, and the government [8]. Both datasets include information on inpatient admissions, with details such as the patient's age, gender, procedures during the admission, and diagnoses coded at discharge. The datasets cover different populations (although not mutually exclusive), and are therefore not directly comparable.

The PHI dataset contains medical and hospital claims made to 13 Australian insurance funds, as well as the Hospital Casemix Protocol data, sent from hospitals to these insurance funds after a patient's discharge and used by the Australian government and insurance industry [9]. Clinical coders record medical services using the Medicare Benefits Scheme (MBS) item numbers (if claimed by a clinician to the insurance fund) and the Australian Classification of Health Interventions (ACHI). Within the public hospital dataset, this MBS code is not present since clinicians are not paid per service use. Both datasets have diagnosis information coded using the International Classification of Diseases-10th revision-Australian Modification (ICD-10-AM).

Clinical information used in forming the diagnosis, such as results of pathology tests or imaging, is not available in either dataset. Prescribing information and pathology requests are also not included.

\section{Criteria for whether a recommendation can be measured}

We developed four criteria to consistently and transparently select recommendations measurable in the two datasets used in our work. The specific criteria and their applied order exclude the least relevant (to the dataset) recommendations first, leaving those recommendations requiring further investigation and resources to determine their measurability.

i. Observable in setting The recommendation is relevant to the dataset and its health care setting.
We excluded recommendations related to procedures or services unlikely to occur in an inpatient setting, such as those usually performed in general practice.

ii. Record of service in claim The service can be recorded in the claim as the relevant and specific code exists for it.

Australian inpatient claims include procedures but not the prescribing of medicines. Hence, recommendations related to in-hospital prescription drugs are not measurable in claims data.

iii. Indication It is possible to map the appropriate/inappropriate patient criteria for a service described in the recommendation to the information within the claim.

Sometimes the caveats on the recommended inappropriate use of a service are too non-specific compared with the clinical detail recorded in the claims. For example, we cannot investigate a recommendation of the form "do not do ... without careful consideration." A CW Australia recommendation states that inguinal hernia repair should not occur: "...without careful consideration, particularly in patients who have significant co-morbidities" [10]. A direct measure of low-value care based on this recommendation would arguably have poor specificity, because it would label too many appropriate inguinal hernia repairs as low-value.

iv. Consistent documentation The service is consistently recorded in the claim according to standard coding practices.

We cannot measure a recommendation across a population if the service is not consistently documented during the data collection, as the service count will be underreported. In some cases, a radiologist will claim for an imaging service for an admitted patient, so the relevant MBS item will be in the private claims dataset. However, the Australian Coding Standards for ACHI coding classify imaging services as 'procedures not normally coded', because "they are usually routine in nature, performed for most patients and/or can occur multiple times in an episode" [11]. Some low-value care described by these recommendations might be identifiable, and so these measures might be of interest to payers, but they are not generalisable because the estimates would be under-representative and possibly biased. 


\section{Selecting measurable recommendations in hospital admissions data}

We investigated 824 recommendations from $\mathrm{CW}$ lists in the US, Canada, Australia, and the UK, downloaded in January 2017 [12-15]. Two authors (KC, TBP) reviewed the recommendations independently to assess measurability in the private and public datasets, and then resolved discrepancies by consensus. Figure 1 illustrates this review process according to the measurement criteria.

\section{Adapting recommendations for measurement}

We followed an approach used by Schwartz et al. [16] and further described in Brett et al. [17], and defined a narrow indicator (more specific, which might exclude some low-value care) and a broad indicator (more sensitive, which might include some appropriate care) for each service when necessary. Where they exist, we used previously published indicators of these low-value services as a starting point $[16,18]$ and adapted these based on the details in our data and health care setting, or modified them in response to clinical advice or updates to recommendations.

Even when a recommendation was measureable according to the criteria there could still be ambiguity on how to define low-value service use. For example, multiple recommendations relating to the same service were sometimes slightly different, such as two CW US recommendations on inferior vena caval filter use from the Society for Vascular Surgery and the American Society of Hematology.

For some indicators, we developed a proxy measure for the "asymptomatic" patient groups. We excluded patients with diagnoses related to possible symptoms (see definitions for carotid endarterectomy and endovascular repair of abdominal aortic aneurysms in Table 1).

When developing the narrow indicator we used the most restrictive of any duplicate or similar recommendations, excluded procedures or diagnoses where appropriateness was unclear, and defined proxy measures to err towards counting care as appropriate. To

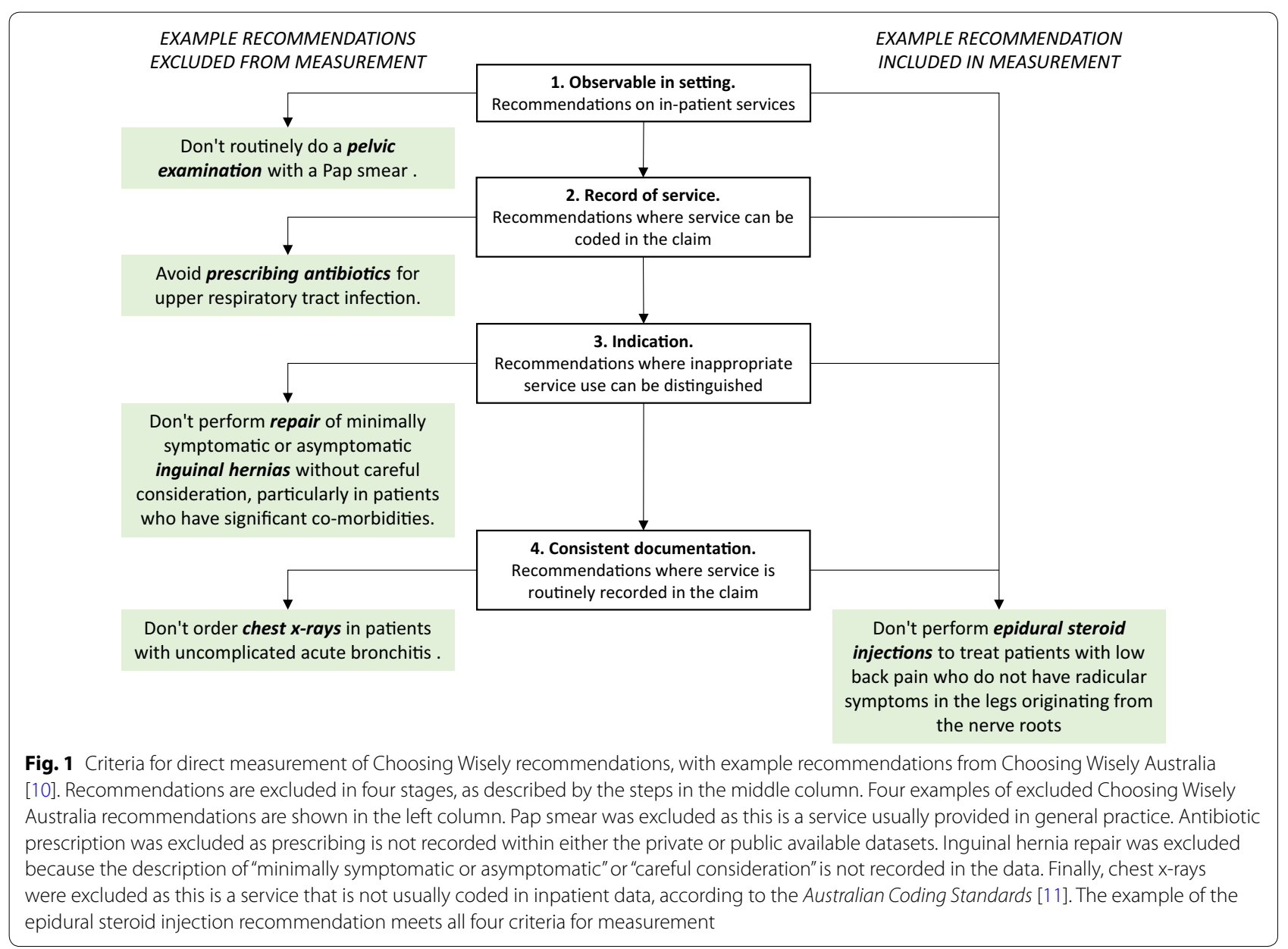




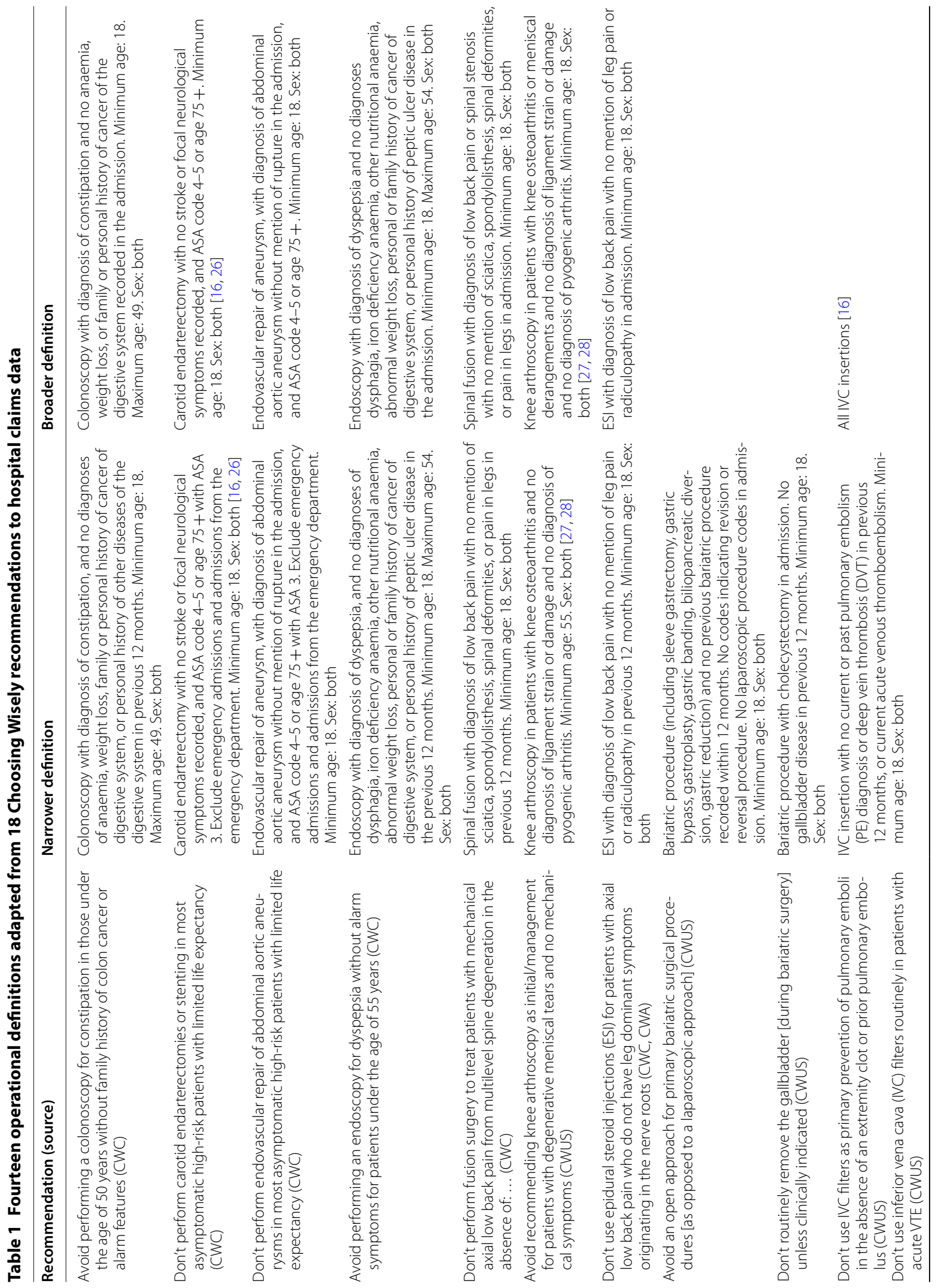




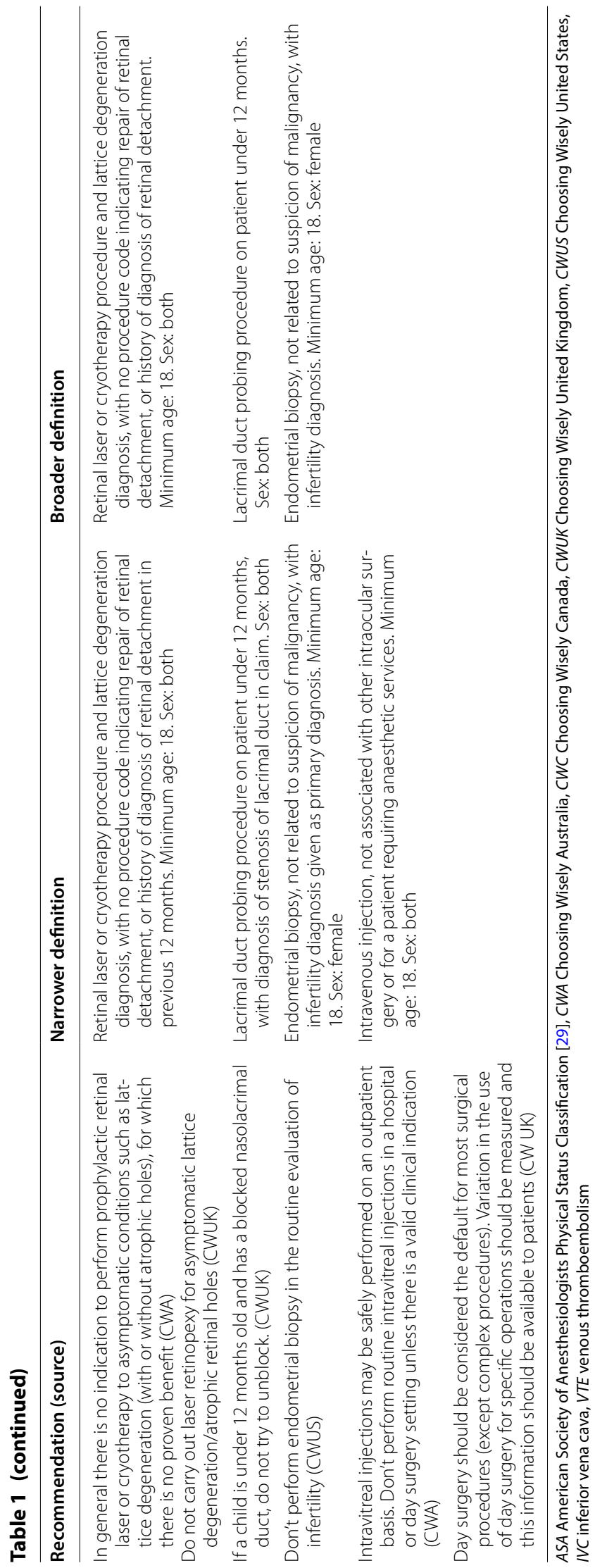

develop the broader definition we included any ambiguous diagnosis or procedure codes that may capture inappropriate care, or adjusted the age, sex, or indications for which the service is potentially low-value.

\section{Clinical review}

Draft indicators were presented at a workshop involving 27 clinicians. Two authors (TBP and AGE) presented the overall approach, and invited feedback on the project as a whole. The clinicians then separated into groups where each reviewed 3 to 4 draft indicators. The clinicians were asked to take the CW recommendations as given (thus respecting the various $\mathrm{CW}$ processes for developing recommendations), and assess whether the narrow and broad indicators adequately captured the care targeted by the recommendation. They examined both the description of the indicator (as presented in Table 1) and the codes used in identifying low-value claims. We revised the indicators to incorporate the feedback, and a clinical coder reviewed the revised versions to assess the codes. Clinical co-authors (JB, IAS) resolved further questions about the indicators, or we occasionally consulted other specialists, including those present at the workshop.

\section{Results}

We excluded 283 recommendations (34.3\% of total) that were not observable in setting as they did not relate to inpatient services (Fig. 2). Then we excluded 203 (37.5\%) of the remaining recommendations from measurement in the PHI data and 231 (42.7\%) in the public hospital data, because there was no record of service in the datasets. These were mainly on pathology or prescribing services. The number excluded in the public and private hospital data differs because some services relate to an MBS item but not an ACHI code. For example, MBS items record inpatient intravitreal injections in PHI claims but not claims from public hospitals (and there is no specific ACHI code for this service).

Of the remaining recommendations, 93 (27.5\%) met the indication criteria in the PHI data and 89 (28.7\%) in the public hospital data, meaning we could identify a low-value indication for the service using the clinical details in the datasets.

Finally, we excluded 75 (81\%) recommendations from the PHI data because the service was not consistently documented in the claims. We also excluded 72 (82\%) from measurement in the public hospital data. This left 18 and 17 measurable recommendations in the PHI and public hospital datasets respectively (2.06 and $1.94 \%$ of all recommendations). Throughout this selection process, we resolved discrepancies for 68 recommendations $(8.25 \%$ of all). The disagreement usually related to whether the 
recommendation was relevant in an inpatient setting or if the low-value indication was measurable.

We adapted 17 of the 18 measurable recommendations into indicators. Three pairs of similar or identical recommendations were combined into single indicators (Table 1). We omitted a UK recommendation that most surgical procedures should be a day surgery and "variation in the use of day surgery for specific operations should be measured". Although it is measurable, adapting this specific recommendation will require considerable input from practitioners for exclusion criteria, depend on regional health systems' day surgery policies, and could include many procedures. We therefore developed 14 indicators of low-value care.

During the clinical review process, participants suggested diagnoses justifying the procedure, thus making the indicators more specifically targeted at low-value care. They also advised against a strict age limit for "limited life expectancy", resulting in a proxy measure based on age and American Society of Anesthesiologists risk score.

\section{Limitations}

Eighteen of the 824 original recommendations were measurable in the datasets after excluding 807 (97.9\%) recommendations. Similar studies have also found that most recommendations are not measurable in routinely collected data. Duckett et al. [18] identified 5 out of 1208 (0.4\%) low-value services they could measure in hospital data. In primary care data, Sprenger et al. [19] measured 34 (2\%) of 1658 recommendations. The small number of measurable recommendations is a consequence of the limited clinical information and health care setting coverage of these datasets (e.g. non-linked inpatient, primary care, pathology, radiology and pharmaceutical data). Using linked data may mean more recommendations are measurable in routinely collected data.

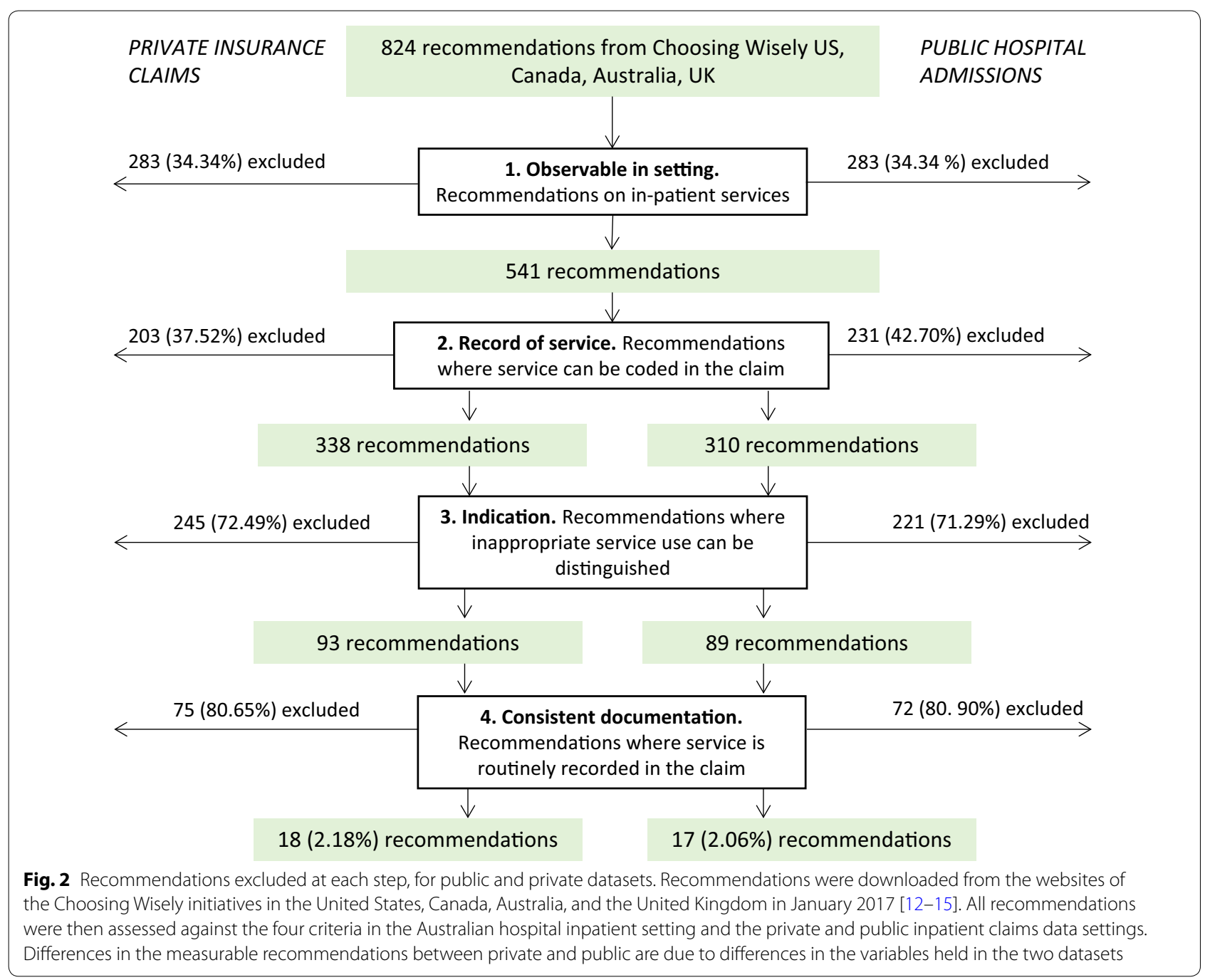


Our operational definitions of low-value care would benefit from further validation, such as comparison to a clinical chart review. This is a common issue when measuring low-value care in routinely collected data [20]. Validation studies using clinical chart review have, however, suggested either reasonable agreement or a conservative estimate of low-value care using the routine data approach [21-23].

These indicators are useful for examining variation and trends and to prioritise low-value care initiatives. $\mathrm{CW}$ is clinician-led and aims to facilitate discussions on appropriate care between doctors and patients, and not to proscribe services $[24,25]$. Large questions still exist around the utility of auditing and feeding back low-value care measurement data to hospital managers and clinicians, recognising there is potential risk to the goodwill underlying Choosing Wisely type initiatives [24]. These indicators are useful, however, in facilitating questions and discussions regarding the true extent of low-value care, particularly where rates appear high within particular settings.

Our criteria allow systematic and transparent selection of recommendations for direct measurement within our data setting. The criteria and approach can be replicated in other data settings, including outside Australia, as well as other inappropriate care recommendations.

\footnotetext{
Abbreviations

ACHI: Australian Classification of Health Intervention; ASA: American Society of Anesthesiologists; CT: computed tomography; CW: Choosing Wisely; CWA: Choosing Wisely Australia; CWC: Choosing Wisely Canada; CWUS: Choosing Wisely United States; DVT: deep vein thrombosis; MBS: Medical Benefits Scheme; NSW: New South Wales; PE: pulmonary embolism; PHI: private health insurance; UK: United Kingdom; US: United States; VTE: venous thromboembolism.
}

\section{Authors' contributions}

KC and TBP assessed the measurability of the Choosing Wisely recommendations, adapted recommendations for measurement, and jointly drafted the paper. IAS and JB provided clinical input for adapting the recommendations. SAP and AGE provided overall supervision and direction to the project and assisted with drafting the paper. All authors read and approved the final manuscript.

\section{Author details}

${ }^{1}$ Menzies Centre for Health Policy, School of Public Health, Charles Perkins Centre, University of Sydney, Sydney, NSW 2006, Australia. ${ }^{2}$ Health Market Quality Program, Capital Markets Cooperative Research Centre, Sydney, NSW 2000, Australia. ${ }^{3}$ Medicines Policy Research Unit, Centre for Big Data Research in Health, University of New South Wales, Level 1, AGSM Building, Sydney, NSW 2052, Australia. ${ }^{4}$ Princess Alexandra Hospital, Brisbane, QLD 4102, Australia.

${ }^{5}$ University of Queensland, Brisbane, QLD 4072, Australia.

\section{Acknowledgements}

We thank the 27 participants of the clinical workshop who reviewed the related methods and indicators used for the hospital data.

\section{Competing interests}

AGE receives salary support as the HCF Research Foundation Professorial Research Fellow, is a Ministerial appointee to the (Australian) Medicare Benefits Schedule (MBS) Review Taskforce, a member of the Choosing Wisely Australia advisory group, the Choosing Wisely International Planning Committee, the ACSQHC's Atlas of Healthcare Variation Advisory Group, and a Board Member of the NSW Bureau of Health Information (BHI). KC and TBP receive salary support via a doctoral scholarship from the Capital Markets Cooperative Research Centre-Health Market Quality Program.

\section{Availability of data and materials}

The datasets generated and/or analysed during the current study are not publicly available for reasons of commercial confidentiality.

\section{Consent for publication}

Not applicable.

\section{Ethics approval and consent to participate}

The University of Sydney Human Research Ethics Committee (Project ID 2015/662) approved the study with respect to the private health insurance dataset, and the NSW Population and Health Services Research Ethics Committee (2015/09/607) approved the study with respect to the public hospital data set.

\section{Funding}

This work is supported by an NHMRC Project Grant (APPID: 1109626), the NHMRC Centre for Research Excellence in Medicines and Ageing (APPID: 1060407). JB receives an NHMRC Postgraduate Scholarship (APPID: 1094304). AGE receives salary support as the HCF Research Foundation Professorial Research Fellow. KC and TBP receive salary support via a doctoral scholarship from the Capital Markets Cooperative Research Centre under the Health Market Quality Program and industry partners Hospital and Medical Benefits System, Ltd, the NSW Ministry of Health, as well as The University of Sydney. KC also receives support from an Australian Government Research Training Program Scholarship. TBP also receives salary support through a University Postgraduate Award from the University of Sydney.

\section{Publisher's Note}

Springer Nature remains neutral with regard to jurisdictional claims in published maps and institutional affiliations.

Received: 16 January 2018 Accepted: 24 February 2018

Published online: 05 March 2018

\section{References}

1. Brownlee S, Chalkidou K, Doust J, Elshaug AG, Glasziou P, Heath I, Nagpal S, Saini V, Srivastava D, Chalmers K, Korenstein D. Evidence for overuse of medical services around the world. Lancet. 2017. https://doi.org/10.1016/ S0140-6736(16)32585-5.

2. Gnjidic D, Elshaug AG. De-adoption and its 43 related terms: harmonizing low-value care terminology. BMC Med. 2015;13:273. https://doi. org/10.1186/s12916-015-0511-4.

3. Elshaug AG, McWilliams JM, Landon BE. The value of low-value lists. JAMA. 2013;309:775-6.

4. Bhatia RS, Levinson W, Shortt S, Pendrith C, Fric-Shamji E, Kallewaard M, Peul W, Veillard J, Elshaug A, Forde I, Kerr EA. Measuring the effect of Choosing Wisely: an integrated framework to assess campaign impact on low-value care. BMJ Qual Saf. 2015;24:523-31.

5. Scott IA, Duckett SJ. In search of professional consensus in defining and reducing low-value care. Med J Aust. 2015;203:179-81.

6. Miller G, Rhyan C, Beaudin-Seiler B, Hughes-Cromwick P. A framework for measuring low-value care. Value Health. 2017. https://doi.org/10.1016/j. jval.2017.10.017.

7. Chalmers K, Pearson S-A, Elshaug AG. Quantifying low-value care: a patient-centric versus service-centric lens. BMJ Qual Saf. 2017;26:855-8.

8. Krassnitze $\mathrm{L}$, Willis $\mathrm{E}$. The public health sector and medicare. In: Willis $\mathrm{E}$, Keleher H, Reynolds L, editors. Understanding the Australian Health Care System. 3rd ed. Australia: Elsevier Health Sciences APAC; 2016. 
9. Srinivasan $U$, Arunasalam B. Leveraging big data analytics to reduce healthcare costs. IT Prof. 2013;15:21-8.

10. NPS MedicineWise: tests, treatments, and procedures for healthcare providers and consumers to question. 2016. http://www.choosingwisely. org.au/recommendations. Accessed 3 Mar 2017.

11. Australian Consortium for Classification Development. Australian coding standards. 9th ed. Wollongong: Australian Consortium for Classification Development; 2015.

12. Choosing Wisely UK. Choosing Wisely UK. 2017. http://www.choosingwi sely.co.uk/i-am-a-clinician/recommendations/. Accessed 20 Sept 2017.

13. ABIM Foundation. Choosing Wisely. 2017. http://www.choosingwisely. org/. Accessed 20 Sept 2017.

14. NPS MedicineWise. Choosing Wisely Australia. 2016. http://www.choos ingwisely.org.au. Accessed 20 Sept 2017.

15. University of Toronto, Canadian Medical Association, St Michael's Choosing Wisely Canada. 2017. https://choosingwiselycanada.org/. Accessed 20 Sept 2017.

16. Schwartz A, Landon B, Elshaug A, Chernew M, McWilliams M. Measuring low-value care in medicare. JAMA Intern Med. 2014;174:1067-76.

17. Brett J, Elshaug AG, Bhatia RS, Chalmers K, Badgery-Parker T, Pearson S-A. A methodological protocol for selecting and quantifying low-value prescribing practices in routinely collected data: an Australian case study. Implement Sci. 2017;12:58. https://doi.org/10.1186/s13012-017-0585-9.

18. Duckett SJ, Breadon P, Romanes D. Identifying and acting on potentially inappropriate care. Med J Aust. 2015;203:183.

19. Sprenger M, Robausch M, Moser A. Quantifying low-value services by using routine data from Austrian primary care. Eur J Pub Health. 2016;26:912-6.

20. de Vries EF, Struijs JN, Heijink R, Hendrikx RJ, Baan CA. Are low-value care measures up to the task? A systematic review of the literature. BMC Health Serv Res. 2016:16:405.

21. Saini SD, Powell AA, Dominitz JA, Fisher DA, Francis J, Kinsinger L, Pittman KS, Schoenfeld P, Moser SE, Vijan S. Developing and testing an electronic measure of screening colonoscopy overuse in a large integrated healthcare system. J Gen Intern Med. 2016;31:53-60.
22. Maier B, Wagner K, Behrens S, Bruch L, Busse R, Schmidt D, Schühlen H, Thieme $\mathrm{R}$, Theres $\mathrm{H}$. Comparing routine administrative data with registry data for assessing quality of hospital care in patients with myocardial infarction using deterministic record linkage. BMC Health Serv Res. 2016;16:605. https://doi.org/10.1186/s12913-016-1840-5.

23. Avoundjian T, Gidwani R, Yao D, Lo J, Sinnott P, Thakur N, Barnett PG. Evaluating two measures of lumbar spine MRI overuse: administrative data versus chart review. J Am Coll Radiol. 2016;13:1057-66. https://doi. org/10.1016/j.jacr.2016.04.013.

24. Wolfson D, Suchman A. Choosing Wisely (R): a case study of constructive engagement in health policy. Healthcare. 2016;4:240-3.

25. Levinson W, Kallewaard M, Bhatia RS, Wolfson D, Shortt S, Kerr EA, Burgers J, Cucic C, Daniels M, Forde I, et al. 'Choosing Wisely': a growing international campaign. BMJ Qual Saf. 2014. https://doi.org/10.1136/bmjgs -2014-003821.

26. EVOLVE. Australian and New Zealand Association of Neurologists Top 5 low-value practices and interventions. 2016. http://evolve.edu.au/ published-lists/australian-and-new-zealand-association-of-neurologists. Accessed 1 Mar 2017

27. Australian Orthopaedic Association. Position Statement from the Australian Knee Society on Arthroscopic Surgery of the Knee, with particular reference to the presence of Osteoarthritis. 2016. http://www.kneesociet y.org.au/resources/aks-arthroscopy-position-statement.pdf. Accessed 2 Mar 2017.

28. National Institute for Health and Care Excellence. Do Not Do Recommendation. 2014. https://www.nice.org.uk/donotdo/do-not-refer-for-arthr oscopic-lavage-and-debridement-as-part-of-treatment-for-osteoarthritisunless-the-person-has-knee-osteoarthritis-with-a-clear-history-of-mecha nical-locking-as-opposed-to-morning. Accessed 1 Mar 2017.

29. Daabiss M. American society of anaesthesiologists physical status classification. Indian J Anaesth. 2011;55:111-5. https://doi.org/10.4103/00195049.79879.

\section{Submit your next manuscript to BioMed Central and we will help you at every step:}

- We accept pre-submission inquiries

- Our selector tool helps you to find the most relevant journal

- We provide round the clock customer support

- Convenient online submission

- Thorough peer review

- Inclusion in PubMed and all major indexing services

- Maximum visibility for your research

Submit your manuscript at www.biomedcentral.com/submit
O Biomed Central 